\title{
Distribución y representatividad de las especies del género Crotalus en las áreas naturales protegidas de México
}

\section{Distribution and representation of Crotalus species within the natural protected areas of Mexico}

\author{
Dinorah Malinaly Paredes-García ${ }^{1}$, Aurelio Ramírez-Bautista ${ }^{1 *}$ y Miguel Angel Martínez-Morales ${ }^{2}$ \\ ${ }^{1}$ Laboratorio de Ecología de Poblaciones, Centro de Investigaciones Biológicas, Universidad Autónoma del Estado de Hidalgo. Apartado postal 1-69, \\ Plaza Juárez, 42001 Pachuca, Hidalgo, México. \\ ${ }^{2}$ Sección Hidalgo, Sociedad para el Estudio y Conservación de las Aves en México, A.C. Francisco I. Madero 58, 43560 Omitlán, Hidalgo, México. \\ *Correspondencia: aurelior@uaeh.edu.mx
}

\begin{abstract}
Resumen. Se realizó un análisis sistemático de la distribución de las especies del género Crotalus en México para conocer los sitios de mayor concentración y evaluar su nivel de representatividad dentro del esquema federal de áreas naturales protegidas (ANP). A partir de registros georreferenciados se construyeron los modelos de distribución potencial de las especies con un número suficiente de registros, empleando los algoritmos GARP y MaxEnt. Con estos mapas de distribución se dedujeron las áreas de mayor concentración de especies y se estimó su presencia en las ANP. La mayor riqueza de especies se concentra en las regiones de la sierra Madre Occidental, el noroeste del Altiplano Mexicano, la porción norte de la sierra Madre Oriental y el extremo oeste de la Faja Volcánica Transmexicana. Se estima que el $84.4 \%$ de las ANP incluyen al menos 1 especie de Crotalus y que el $88.2 \%$ de las especies que se distribuyen en México están presentes al menos dentro de 1 ANP. En este contexto de alta representatividad, el plantear la incorporación de ANP adicionales para la protección y conservación de crotalinos se dificulta, por lo que debe optarse por estrategias alternativas, tales como educación ambiental, conservación de hábitats mediante el uso sustentable de recursos, y el manejo y reproducción en cautiverio de especies en riesgo; además, se debe fomentar la investigación y el monitoreo de poblaciones.
\end{abstract}

Palabras clave: Crotalinae, Viperidae, riqueza de especies, modelos de distribución, GARP, MaxEnt, protección, conservación, manejo.

\begin{abstract}
We performed a systematic analysis of the distribution of the Crotalus species in Mexico to depict the places of high species concentration and to evaluate its representation within the federal natural protected areas (NPAs) scheme. Based on geo-referenced records, we built distribution models for species with enough records using the GARP and MaxEnt algorithms. We then deduced areas of high species concentration and estimated its presence in the NPAs. The regions with the highest species richness are the Sierra Madre Occidental, the northwestern Mexican Highlands, the northern portion of the Sierra Madre Oriental, and the western most portion of the Transmexican Volcanic Belt. We estimated that $84.4 \%$ of the NPAs include at least 1 species and $88.2 \%$ of the species distributed in Mexico occur in at least 1 NPA. In this context of high representation, the idea of incorporating additional NPAs for the protection and conservation of crotalines is difficult; thus, alternative strategies should be considered such as environmental awareness, habitat conservation through the sustainable use of resources, and captive management and breeding of endangered species. Research and population monitoring should also be promoted.
\end{abstract}

Key words: Crotalinae, Viperidae, species richness, distribution models, GARP, MaxEnt, protection, conservation, management.

\section{Introducción}

El género Crotalus, perteneciente a la familia Viperidae, es un grupo de serpientes altamente representativo en México; lo componen alrededor de 37 especies, de las cuales $34(92 \%)$ se distribuyen en México, siendo

Recibido: 28 agosto 2010; aceptado: 20 julio 2010 endémicas 22 de éstas (Flores-Villela, 1993; Grismer, 2002; Alvarado-Díaz y Campbell, 2004; Campbell y Lamar, 2004; Flores-Villela y Canseco-Márquez, 2004; Campbell y Flores-Villela, 2008). Se estima que la diversidad más alta de este género está en el norte del país, en las cadenas montañosas de la sierra Madre Occidental y en el Altiplano Mexicano (Campbell y Lamar, 1989, 2004).

Los miembros de este género ocupan varios tipos de vegetación, principalmente los bosques de pino-encino y 
encino, el matorral xerófilo y el bosque mesófilo de montaña. Además, estas especies pueden encontrarse desde el nivel del mar hasta arriba de los $4000 \mathrm{~m}$ (Campbell y Lamar, 1989, 2004).

El género Crotalus es un grupo de gran interés desde diversos puntos de vista, a pesar de estar constituido por especies que no siempre son carismáticas por ser altamente venenosas. Por ejemplo, estas especies forman parte de tramas tróficas y pueden actuar como control biológico de poblaciones de especies consideradas plaga, como las de algunos roedores (Campbell y Lamar, 2004); asimismo, culturalmente tienen o han tenido un importante uso y simbolismo, como en el caso de las culturas prehispánicas (Castellón-Huerta, 2001; Jáuregui, 2001; Taube, 2001).

Con base en la normatividad mexicana vigente (SEMARNAT, 2010), de las 34 especies de Crotalus que se distribuyen en México, 1 (C. transversus), que además es endémica, se encuentra en la categoría "en peligro de extinción"; 7, también endémicas, en la de"amenazada", y 16 en "sujeta a protección especial". Asimismo, de acuerdo con el sistema de categorización de la IUCN (2010), C. catalinensis está considerada como "critically endangered", C. pusillus como "endangered" y $C$. stejnegeri como "vulnerable", todas éstas, endémicas de México.

A la fecha, no se ha realizado un análisis completo y sistemático de la distribución de las especies del género Crotalus en México, ni tampoco de su nivel de representatividad dentro del esquema mexicano de áreas naturales protegidas (ANP), a pesar de su importancia biológica, ecológica, cultural y de salud pública, y de que muchas de ellas se encuentran en un estado delicado de conservación. Así, resulta imprescindible realizar un estudio riguroso para conocer con mayor precisión su distribución y evaluar la efectividad de las ANP en México, para asegurar su conservación a largo plazo. En el presente estudio se busca cubrir este vacío de información y coadyuvar en el planteamiento de estrategias de manejo para la conservación de los crotalinos en el país.

\section{Materiales y métodos}

Fuentes de información. Se recabaron registros geográficos de las especies del género Crotalus disponibles en una base de datos de anfibios y reptiles de México construida por el segundo autor a partir de registros en colecciones científicas nacionales y extranjeras (ParedesGarcía, 2008). De esta base de datos, se obtuvieron 1157 registros de 21 de las 34 especies del género Crotalus que se distribuyen en México (Cuadro 1). Con el fin de obtener registros adicionales, se consultó la base de datos de la Global Biodiversity Information Facility (GBIF, 2008). De ésta se obtuvo información de 11 especies del género Crotalus y a partir de la información de los sitios de colecta, se derivaron las coordenadas geográficas de cada registro. En el caso de las especies $C$. tancitarensis y $C$. ericsmithi, descritas recientemente, y de las que no se obtuvieron registros en las bases de datos antes mencionadas, se consultaron directamente las publicaciones de sus descripciones (Alvarado-Díaz y Campbell, 2004; Campbell y Flores-Villela, 2008).

Modelado de la distribución potencial. En la estimación de la distribución potencial de las especies de Crotalus se empleó el algoritmo GARP (Genetic Algorithm of Rule-Set Prediction) (Stockwell y Noble, 1992; Stockwell y Peters, 1999), disponible en el programa DesktopGARP v. 1.1.6 (Scachetti-Pereira, 2002), y el algoritmo MaxEnt (Maximum Entropy) (Phillips et al., 2006). Estos algoritmos modelan el nicho ecológico de especies mediante la relación entre los puntos de presencia conocidos de la especie y las variables ambientales incluidas en el modelo; a partir de esta relación, el algoritmo extrapola la presencia de la especie a las áreas donde se desconoce su presencia o ausencia (Lindermayer et al., 1996). Con estos algoritmos, se generaron los modelos de distribución potencial de 23 especies (Cuadro1), para las cuales los registros geográficos fueron suficientemente representativos. En la construcción de los modelos, se emplearon las capas digitales de clima actual, pendiente, aspecto y altitud (Hijmans et al., 2004, 2005).

En el caso de GARP, se generaron 100 modelos por especie con un límite de convergencia de 0.1 y un número máximo de 1000 iteraciones; asimismo, se emplearon los 4 tipos de reglas (atómica, distribución, distribución negada y regresión logística), un umbral de omisión duro (hard) con un $10 \%$ de error de omisión en los modelos generados y un umbral de error de comisión del 50\%. Se utilizó la opción best subset selection para seleccionar el subconjunto de los 10 mejores modelos en los que se repitió el patrón de áreas de distribución de manera más estable (Stockwell y Peters, 1999; Anderson et al., 2003). Posteriormente, en un sistema de información geográfica (SIG, ArcView 3.3), fueron sobrepuestos los mejores modelos de cada especie para obtener un mapa de consenso. Se usó el criterio de $\geq 50 \%$ de coincidencia entre modelos para obtener un mapa de presencia-ausencia. Con el fin de hacer más precisa la estimación de la distribución potencial de cada especie, se seleccionaron las áreas de coincidencia entre la distribución estimada de la especie y los tipos de vegetación que utiliza, de acuerdo con la información de su historia natural en Campbell y Lamar (2004). En este 
Cuadro 1. Especies del género Crotalus registradas para México*

\begin{tabular}{|c|c|c|c|c|c|}
\hline \multirow[t]{2}{*}{ Especie } & \multirow{2}{*}{$\begin{array}{c}\text { Número de registros } \\
\text { geográficos } \\
\text { independientes }\end{array}$} & \multirow[t]{2}{*}{ Método } & \multirow[t]{2}{*}{ Endemismo } & \multicolumn{2}{|c|}{$\begin{array}{c}\text { Esquema de categorización de } \\
\text { riesgo }\end{array}$} \\
\hline & & & & $\begin{array}{l}\text { SEMARNAT } \\
\quad(2010)\end{array}$ & $\begin{array}{l}I U C N^{2} \\
(2010)\end{array}$ \\
\hline C. angelensis & 2 & SIG & Endémica & & $\mathrm{LC}$ \\
\hline C. aquilus & 12 & GARP & Endémica & $\operatorname{Pr}$ & $\mathrm{LC}$ \\
\hline C. atrox & 201 & GARP & No endémica & $\operatorname{Pr}$ & $\mathrm{LC}$ \\
\hline C. basiliscus & 114 & GARP & Endémica & $\operatorname{Pr}$ & $\mathrm{LC}$ \\
\hline C. caliginis & 17 & SIG & Endémica & & $\mathrm{LC}$ \\
\hline C. catalinensis & 9 & SIG & Endémica & A & $\mathrm{CR}$ \\
\hline C. cerastes & 57 & MaxEnt & No endémica & $\operatorname{Pr}$ & $\mathrm{LC}$ \\
\hline C. enyo & 54 & GARP & Endémica & A & $\mathrm{LC}$ \\
\hline C. ericsmithi & 1 & SIG & Endémica & & \\
\hline C. estebanensis & 5 & SIG & Endémica & & $\mathrm{LC}$ \\
\hline C. intermedius & 12 & MaxEnt & Endémica & $\mathrm{A}$ & $\mathrm{LC}$ \\
\hline C. lannomi & 2 & SIG & Endémica & A & DD \\
\hline C. lepidus & 119 & GARP & No endémica & $\operatorname{Pr}$ & $\mathrm{LC}$ \\
\hline C. lorenzoensis & 1 & SIG & Endémica & & $\mathrm{LC}$ \\
\hline C. mitchelli & 29 & GARP & No endémica & $\operatorname{Pr}$ & $\mathrm{LC}$ \\
\hline C. molossus & 147 & GARP & No endémica & $\operatorname{Pr}$ & $\mathrm{LC}$ \\
\hline C. muertensis & 1 & SIG & Endémica & & $\mathrm{LC}$ \\
\hline C. oreganus & 11 & GARP & No endémica & & $\mathrm{LC}$ \\
\hline C.polystictus & 46 & MaxEnt & Endémica & $\operatorname{Pr}$ & $\mathrm{LC}$ \\
\hline C.pricei & 90 & GARP & No endémica & $\operatorname{Pr}$ & $\mathrm{LC}$ \\
\hline C. pusillus & 24 & MaxEnt & Endémica & A & $\mathrm{EN}$ \\
\hline C. ravus & 149 & GARP & Endémica & A & $\mathrm{LC}$ \\
\hline C. ruber & 21 & MaxEnt & No endémica & $\operatorname{Pr}$ & $\mathrm{LC}$ \\
\hline C. scutulatus & 119 & GARP & No endémica & $\operatorname{Pr}$ & LC \\
\hline C. simus & 44 & GARP & No endémica & $\operatorname{Pr}$ & \\
\hline C. stejnegeri & 4 & MaxEnt & Endémica & A & VU \\
\hline C. tancitarensis & 1 & SIG & Endémica & & $\mathrm{DD}$ \\
\hline C. tigris & 16 & MaxEnt & No endémica & $\operatorname{Pr}$ & $\mathrm{LC}$ \\
\hline C. tortugensis & 10 & SIG & Endémica & $\operatorname{Pr}$ & $\mathrm{LC}$ \\
\hline C. totonacus & 12 & GARP & Endémica & $\operatorname{Pr}$ & \\
\hline C. transversus & 11 & SIG & Endémica & $\mathrm{P}$ & $\mathrm{LC}$ \\
\hline C. triseriatus & 133 & GARP & Endémica & & $\mathrm{LC}$ \\
\hline C. viridis & 11 & GARP & No endémica & $\operatorname{Pr}$ & $\mathrm{LC}$ \\
\hline C. willardi & 14 & MaxEnt & No endémica & $\operatorname{Pr}$ & $\mathrm{LC}$ \\
\hline
\end{tabular}

* Fuentes: Flores-Villela (1993), Grismer (2002), Alvarado-Díaz y Campbell (2004), Campbell y Lamar (2004), Flores-Villela y Canseco-Márquez (2004) y Campbell y Flores-Villela (2008).

${ }^{1} \mathrm{P}$, en peligro de extinción; A, amenazada; Pr, sujeta a protección especial.

${ }^{2} \mathrm{CR}$, critically endangered; EN, endangered; VU, vulnerable; LC, least concern; DD, data deficient. 
Cuadro 2. Áreas Naturales Protegidas con decreto federal donde se estima que hay al menos una especie del género Crotalus

\begin{tabular}{|c|c|c|}
\hline$A N P^{l}$ & Estado & Especies de Crotalus \\
\hline APFF Ajos/Bavispe & Son & atrox, lepidus, molossus, pricei, scutulatus, tigris, \\
\hline APFF Bala'an Ka'ax & Q Roo & $\begin{array}{l}\text { viridis } \\
\text { simus }\end{array}$ \\
\hline APFF Boquerón de Tonalá & Oax & molossus, ravus, scutulatus \\
\hline APFF Cabo San Lucas & $\mathrm{BCS}$ & atrox, mitchelli, ruber \\
\hline APFF Campo Verde & Chih, Son & lepidus, molossus, pricei, scutulatus, willardi \\
\hline APFF Cañón de Santa Elena & Chih & atrox, molossus, scutulatus \\
\hline APFF Cascada de Agua Azul & Chis & simus \\
\hline APFF Ciénegas del Lerma & Mex & transversus, triseriatus \\
\hline APFF Corredor Biológico Chichinautzin & $\begin{array}{l}\text { DF, Mor, } \\
\text { Mex }\end{array}$ & $\begin{array}{l}\text { molossus, polystictus, ravus, scutulatus, simus, } \\
\text { transversus, triseriatus }\end{array}$ \\
\hline APFF Cuatrociénegas & Coah & atrox, lepidus, molossus,, scutulatus \\
\hline APFF Chan-Kin & Chis & simus \\
\hline APFF El Jabalí & Col & basiliscus, molossus, polystictus, pusillus, scutulatus \\
\hline APFF Islas del Golfo de California & $\begin{array}{l}\mathrm{BC}, \mathrm{BCS} \text {, } \\
\text { Son, Sin }\end{array}$ & $\begin{array}{l}\text { angelensis, atrox, cerastes, estebanensis, lorenzoensis, } \\
\text { muertensis, ruber, tigris, tortuguensis }\end{array}$ \\
\hline APFF La Primavera & Jal & basiliscus, lepidus, molossus, polystictus, scutulatus \\
\hline APFF Laguna de Términos & Camp & simus \\
\hline APFF Laguna Madre y delta del Río Bravo & Tama & atrox, molossus, totonacus \\
\hline APFF Maderas del Carmen & Coah & atrox, lepidus, molossus, scutulatus \\
\hline APFF Manglares de Nichupté & Q Roo & simus \\
\hline APFF Médanos de Samalayuca & Chih & atrox, molossus \\
\hline APFF Meseta de Cacaxtla & $\operatorname{Sin}$ & atrox, basiliscus, lepidus, molossus, simus \\
\hline APFF Metzabok & Chis & simus \\
\hline APFF Naha & Chis & simus \\
\hline APFF Ocampo & Coah & atrox, lepidus, molossus, scutulatus \\
\hline APFF Otoch Ma'ax Yetel Kooh & Yuc & simus \\
\hline APFF Papigochic & Chih & lepidus, molossus, pricei, scutulatus, willardi \\
\hline APFF Pico de Tancítaro & Mich & basiliscus, molossus, tancitarensis, triseriatus \\
\hline $\begin{array}{l}\text { APFF Sierra de Álamos-Río } \\
\text { Cuchujaqui }\end{array}$ & Chih, Son & basiliscus, lepidus, molossus, pricei, scutulatus, tigris \\
\hline APFF Sierra de Álvarez & SLP & atrox, lepidus, molossus, scutulatus \\
\hline APFF Sierra de Quila & Jal & basiliscus, lepidus, molossus, polystictus, scutulatus \\
\hline APFF Sierra la Mojonera & SLP & atrox, lepidus, molossus, scutulatus \\
\hline APFF Tutuaca & Chih, Son & $\begin{array}{l}\text { basiliscus, lepidus, molossus, pricei, scutulatus, } \\
\text { willardi }\end{array}$ \\
\hline APFF Uaymil & Q Roo & simus \\
\hline APFF Valle de Los Cirios & $\mathrm{BC}$ & atrox, cerastes, enyo, mitchelli, oreganus, ruber \\
\hline APFF Yum Balam & Q Roo & simus \\
\hline $\begin{array}{l}\text { APRN Cuenca Hidrográfica } \\
\text { del Río Necaxa }\end{array}$ & Pue & $\begin{array}{l}\text { aquilus, atrox, intermedius, ravus, scutulatus, } \\
\text { triseriatus }\end{array}$ \\
\hline $\begin{array}{l}\text { APRN Cuencas de los Ríos Valle de } \\
\text { Bravo, Malacatepec, Tilostoc y Temascaltepec }\end{array}$ & Mex, Mich & $\begin{array}{l}\text { molossus, polystictus, ravus, scutulatus, simus, } \\
\text { transversus, triseriatus }\end{array}$ \\
\hline APRN Las Huertas & Col & simus \\
\hline
\end{tabular}


Cuadro 2. Continúa.

\begin{tabular}{|c|c|c|}
\hline$A N P^{l}$ & Estado & Especies de Crotalus \\
\hline MN Bonampak & Chis & simus \\
\hline MN Cerro de la Silla & NL & atrox, lepidus, molossus, scutulatus \\
\hline MN Río Bravo del Norte & Chih, Coah & atrox, lepidus, molossus, scutulatus \\
\hline MN Yagul & Oax & molossus, ravus \\
\hline MN Yaxchilán & Chis & simus \\
\hline PN Archipiélago de San Lorenzo & $\mathrm{BC}$ & cerastes, lorenzoensis, ruber \\
\hline PN Archipiélago Espíritu Santo & $\mathrm{BCS}$ & atrox, ruber \\
\hline PN Arrecife de Puerto Morelos & Q Roo & simus \\
\hline PN Arrecifes de Cozumel & Q Roo & simus \\
\hline PN Arrecifes de Xcalak & Q Roo & simus \\
\hline PN Bahía de Loreto & $\mathrm{BCS}$ & atrox, catalinensis, cerastes, mitchelli, ruber \\
\hline PN Barranca del Cupatitzio & Mich & basiliscus, molossus, triseriatus \\
\hline PN Benito Juárez & Oax & intermedius, molossus, ravus \\
\hline PN Bosencheve & Mex, Mich & $\begin{array}{l}\text { basiliscus, molossus, polystictus, scutulatus, } \\
\text { transversus, triseriatus }\end{array}$ \\
\hline PN Cañón del Río Blanco & Ver, Pue & $\begin{array}{l}\text { intermedius, molossus, ravus, scutulatus, simus, } \\
\text { triseriatus }\end{array}$ \\
\hline PN Cañón del Sumidero & Chis & simus \\
\hline PN Cascada de Bassaseachic & Chih & $\begin{array}{l}\text { basiliscus, lepidus, molossus, pricei, scutulatus, } \\
\text { willardi }\end{array}$ \\
\hline PN Cerro de Garnica & Mich & basiliscus, triseriatus \\
\hline PN Cerro de la Estrella & DF & molossus, polystictus, scutulatus \\
\hline PN Cerro de Las Campanas & Qro & atrox, lepidus, molossus, scutulatus \\
\hline PN Cofre de Perote & Ver & intermedius \\
\hline PN Cumbres de Majalca & Chih & $\begin{array}{l}\text { atrox, lepidus, molossus, pricei, scutulatus, viridis, } \\
\text { willardi }\end{array}$ \\
\hline PN Cumbres de Monterrey & NL, Coah & atrox, lepidus, molossus, scutulatus \\
\hline PN Cumbres del Ajusco & $\mathrm{DF}$ & transversus \\
\hline PN Desierto de los Leones & $\mathrm{DF}$ & transversus \\
\hline PN Desierto del Carmen o de Nixcongo & Mex & molossus, ravus, transversus, triseriatus \\
\hline PN Dzibilchantún & Yuc & simus \\
\hline PN El Chico & Hgo & aquilus, intermedius \\
\hline PN El Cimatario & Qro & atrox, lepidus, molossus, scutulatus \\
\hline PN El Histórico Coyoacán & DF & molossus, polystictus \\
\hline PN El Potosí & SLP & atrox, lepidus, molossus, scutulatus \\
\hline PN El Sabinal & NL & atrox, lepidus, molossus \\
\hline PN El Tepeyac & DF & molossus, polystictus, scutulatus \\
\hline PN El Tepozteco & Mor, DF & molossus, ravus, scutulatus, transversus, triseriatus \\
\hline PN El Veladero & Gro & simus \\
\hline PN Fuentes Brotantes de Tlalpan & $\mathrm{DF}$ & molossus, scutulatus \\
\hline PN General Juan N. Álvarez & Gro & molossus, ravus \\
\hline PN Gogorrón & SLP & atrox, lepidus, molossus, scutulatus \\
\hline
\end{tabular}


Cuadro 2. Continúa

\begin{tabular}{|c|c|c|}
\hline$A N P^{I}$ & Estado & Especies de Crotalus \\
\hline PN Grutas de Cacahuamilpa & Gro, Mor & molossus, ravus, scutulatus, simus \\
\hline PN Huatulco & Oax & simus \\
\hline PN Insurgente José María Morelos & Mich & $\begin{array}{l}\text { basiliscus, lepidus, molossus, polystictus, scutulatus, } \\
\text { transversus, triseriatus }\end{array}$ \\
\hline PN Iztaccihuatl - Popocatepelt & Mex, Pue, & intermedius, polystictus, transversus \\
\hline PN Lago de Camécuaro & Mich & basiliscus, lepidus, molossus, scutulatus \\
\hline PN Lagunas de Chacahua & Oax & simus \\
\hline PN Lagunas de Montebello & Chis & simus \\
\hline PN Lagunas de Zempoala & Mex, Mor & transversus \\
\hline PN Lomas de Padierna & DF & transversus \\
\hline PN Los Mármoles & Hgo & aquilus, atrox, lepidus, molossus, scutulatus \\
\hline PN Los Novillos & Coah & atrox, molossus, scutulatus \\
\hline PN Los Remedios & Mex & molossus, polystictus, scutulatus \\
\hline PN Malinche o Matlalcuéyatl & Tlax, Pue & $\begin{array}{l}\text { intermedius, molossus, polystictus, ravus, scutulatus, } \\
\text { triseriatus }\end{array}$ \\
\hline PN Molino de Flores Netzahualcóyotl & Mex & atrox, molossus, scutulatus \\
\hline PN Nevado de Colima & Jal, Col & basiliscus, molossus, polystictus, pusillus, transversus \\
\hline PN Palenque & Chis & simus \\
\hline PN Pico de Orizaba & Ver, Pue & intermedius \\
\hline PN Rayón & Mich & triseriatus \\
\hline PN Sacromonte & Mex & molossus, transversus \\
\hline PN Sierra de Órganos & $\mathrm{Zac}$ & lepidus, molossus, pricei, scutulatus \\
\hline PN Sierra de San Pedro Mártir & $\mathrm{BC}$ & atrox, enyo, mitchelli \\
\hline PN Sistema Arrecifal Veracruzano & Ver & simus \\
\hline PN Tula & Hgo & atrox, molossus, scutulatus \\
\hline PN Tulum & Q Roo & simus \\
\hline PN Xicoténcatl & Tlax & molossus, polystictus, scutulatus \\
\hline RB Alto Golfo de California y Delta del Río Colorado & BC, Son & cerastes, ruber \\
\hline RB Arrecifes de Sian Ka'an & Q Roo & simus \\
\hline $\begin{array}{l}\text { RB Bahía de los Ángeles, Canales de Ballenas y } \\
\text { Salsipuedes }\end{array}$ & $\mathrm{BC}$ & angelensis, enyo, ruber \\
\hline RB Barranca de Metztitlán & Hgo & $\begin{array}{l}\text { aquilus, atrox, intermedius, lepidus, polystictus, } \\
\text { molossus, scutulatus }\end{array}$ \\
\hline RB Calakmul & Camp & simus \\
\hline RB Chamela-Cuixmala & Jal & basiliscus, simus \\
\hline RB Complejo Lagunar Ojo de Liebre & $\mathrm{BC}, \mathrm{BCS}$ & enyo, mitchelli, ruber \\
\hline RB El Pinacate y Gran Desierto de Altar & Son & atrox, cerastes, tigris \\
\hline RB El Triunfo & Chis & simus \\
\hline RB El Vizcaíno & $\mathrm{BCS}$ & atrox, cerastes, enyo, mitchelli, oreganus, ruber \\
\hline RB Janos & Chih & atrox, lepidus, molossus scutulatus, viridis, willardi \\
\hline RB La Encrucijada & Chis & simus \\
\hline RB La Michilía & Dgo & basiliscus, lepidus, molossus, pricei, scutulatus \\
\hline
\end{tabular}


Cuadro 2. Continúa

\begin{tabular}{|c|c|c|}
\hline$A N P^{l}$ & Estado & Especies de Crotalus \\
\hline RB La Sepultura & Chis, Oax & ravus, simus \\
\hline RB Lacan-Tun & Chis & simus \\
\hline RB Los Petenes & Camp & simus \\
\hline RB Los Tuxtlas & Ver & simus \\
\hline RB Mapimí & Dgo, Chih, & atrox, lepidus, molossus, scutulatus \\
\hline RB Mariposa Monarca & $\begin{array}{l}\text { Coah } \\
\text { Mex, Mich }\end{array}$ & $\begin{array}{l}\text { basiliscus, molossus, polystictus, ravus, scutulatus, } \\
\text { triseriatus }\end{array}$ \\
\hline RB Montes Azules & Chis & simus \\
\hline RB Pantanos de Centla & Tab, Camp & simus \\
\hline RB Ría Celestún & Yuc, Camp & simus \\
\hline RB Ría Lagartos & Q Roo, Yuc & simus \\
\hline RB Selva El Ocote & Chis & simus \\
\hline RB Sian Ka'an & Q Roo & simus \\
\hline RB Sierra de Abra Tanchipa & SLP & atrox, lepidus, molossus, pricei, scutulatus, totonacus \\
\hline RB Sierra de Huautla & Mor, Pue & molossus, ravus, scutulatus, simus, triseriatus \\
\hline RB Sierra de Manantlán & Jal, Col & $\begin{array}{l}\text { basiliscus, lepidus, molossus, polystictus, pusillus, } \\
\text { scutulatus, simus }\end{array}$ \\
\hline RB Sierra Gorda & Qro & $\begin{array}{l}\text { aquilus, atrox, lepidus, molossus, pricei, scutulatus, } \\
\text { totonacus }\end{array}$ \\
\hline RB Sierra Gorda de Guanajuato & Gto & aquilus, atrox, lepidus, molossus, scutulatus \\
\hline RB Sierra La Laguna & $\mathrm{BCS}$ & atrox, enyo, mitchelli, ruber \\
\hline RB Tehuacán-Cuicatlán & Pue, Oax & $\begin{array}{l}\text { intermedius, molossus, ravus, scutulatus, simus, } \\
\text { triseriatus }\end{array}$ \\
\hline RB Tiburón Ballena & Q Roo & simus \\
\hline RB Volcán Tacaná & Chis & simus \\
\hline RB Zicuirán-Infiernillo & Mich & basiliscus, pusillus, simus, triseriatus \\
\hline S Islas de La Bahía de Chamela & Jal & basiliscus, simus \\
\hline S Playa Adyacente a la localidad denominada Río Lagartos & Yuc & simus \\
\hline S Playa Cuitzmala & Jal & atrox \\
\hline S Playa de Escobilla & Oax & simus \\
\hline S Playa de la Bahía de Chacahua & Oax & simus \\
\hline S Playa de Maruata y Colola & Mich & simus \\
\hline S Playa de Mismaloya & Jal & basiliscus, simus \\
\hline S Playa de Rancho Nuevo & Tam & atrox \\
\hline S Playa de Tierra Colorada & Gro & simus \\
\hline S Playa Mexiquillo & Mich & simus \\
\hline S Playa Teopa & Jal & simus \\
\hline
\end{tabular}

${ }^{1}$ APFF, Área de Protección de Flora y Fauna; APRN, Área de Protección de Recursos Naturales; MN, Monumento Natural; PN, Parque Nacional; RB, Reserva de la Biosfera; S: Santuario. 
trabajo, se utilizó el mapa digital de uso de suelo y vegetación elaborado por CONABIO (1999).

En la construcción de los modelos con el algoritmo MaxEnt se empleó el mismo conjunto de variables ambientales y datos de presencia que en GARP. Los mapas de distribución potencial obtenidos mediante MaxEnt, se transformaron a mapas de presencia-ausencia en ArcView 3.3, utilizando un criterio de coincidencia $\geq 0.5$ (Phillips et al., 2006).

Posteriormente, se seleccionó el mejor modelo de distribución potencial para cada especie, generado por uno u otro algoritmo, con base en su distribución conocida y en su historia natural registrada por Grismer (2002) y Campbell y Lamar (2004).

De las 11 especies en las que no fue posible generar los modelos de distribución potencial mediante los algoritmos GARP o MaxEnt, 7 correspondieron a endémicas de las islas de los estados de Baja California y Baja California Sur, y las otras 4 a endémicas continentales con distribución conocida restringida a 1 localidad. En el caso de las especies insulares, los mapas de distribución potencial se construyeron seleccionando las islas correspondientes, con base en las coordenadas geográficas de sus registros y a partir de la información y mapas de Grismer (2002) y Campbell y Lamar (2004). En el caso de las especies continentales, los mapas se elaboraron en un SIG a partir de los datos de colecta, los tipos de vegetación que utilizan, sus rangos de distribución altitudinal y el clima descritos en Grismer (2002), Alvarado-Díaz y Campbell (2004), Campbell y Lamar (2004), y Campbell y Flores-Villela (2008), empleando para ello los mapas digitales de uso de suelo y vegetación (CONABIO, 1999), topografía (CONABIO, 1998) y clima (García y CONABIO, 1998).

Riqueza de especies y representatividad en las ANP. Una vez obtenidos los modelos de distribución potencial de cada una de las 34 especies de Crotalus, se sobrepuso el mapa digital de los polígonos de las ANP con decreto federal (CONANP, 2010) a los modelos de distribución potencial de las especies para determinar qué especies y en cuáles ANP se encuentran presentes. Así, se evaluó el nivel de representatividad de las especies del género Crotalus en las ANP y la efectividad de este esquema de protección en la conservación a largo plazo de las especies de crotalinos.

Para obtener el mapa de riqueza de especies del país, se sumaron en un SIG los 34 modelos de distribución potencial generados. Finalmente, se realizó una correlación entre el número de ANP en las que una determinada especie está incluida y la extensión de su distribución, con la finalidad de saber si esta extensión está relacionada con su representatividad en las ANP.

\section{Resultados}

Distribución y riqueza de especies de Crotalus. En general, no se percibieron diferencias significativas entre los modelos de distribución potencial generados con GARP y MaxEnt para la mayoría de las especies. Asimismo, con ambos algoritmos, los mapas de distribución potencial de las especies de Crotalus que fueron generados coinciden, en términos generales, con los que presentan Grismer (2002) y Campbell y Lamar (2004). Sin embargo, en algunos de los modelos de distribución potencial generados se encontraron inconsistencias importantes; por ejemplo, en zonas del estado de Sonora, se estimó la presencia de especies que hasta el momento no se han registrado. Con base en los resultados de este estudio, en los municipios de Pitiquito, Hermosillo y Carbó se estimó la presencia de las especies $C$. atrox, $C$. cerastes, C. lepidus, C. mitchelli, $C$. molossus y $C$. tigris, de las cuales, la presencia de C. cerastes y C. mitchelli no está registrada (Campbell y Lamar, 2004). Asimismo, en la isla Cozumel, donde ninguna especie se ha registrado, los modelos predicen la presencia de $C$. simus.

En sentido inverso, se presentó otra inconsistencia entre los modelos generados y la distribución conocida de las especies en sitios donde se muestran parches que indican la no presencia de especies de Crotalus, como es el caso de los municipios de Mexicali, Ensenada y Tijuana, en Baja California, así como en los municipios de Puerto Peñasco y San Luis Río Colorado, en Sonora. Según Campbell y Lamar (2004), las especies C. atrox, C. cerastes, C. mitche$l l i, C$. oreganus y $C$. ruber están presentes en estos sitios. Lo mismo ocurre en los municipios de Ascensión, Coyame del Sotol y Juárez, en Chihuahua, donde Campbell y Lamar (2004) indican la presencia de C. atrox, C. molossus y $C$. viridis. De igual forma, en la parte sureste del estado de Hidalgo, Tlaxcala, noreste de Puebla y centro de Veracruz, Campbell y Lamar (2004) y Valencia (2006) señalan la presencia de las especies C. atrox, C. intermedius, C. molossus, C. polystictus, C. ravus, C. scutulatus y C. triseriatus.

Se calcula que la mayor riqueza de especies de Crotalus se presenta en la mitad norte de México, en las regiones correspondientes a la sierra Madre Occidental y el noroeste del Altiplano Mexicano, así como en la porción norte de la sierra Madre Oriental y en el extremo oeste de la Faja Volcánica Transmexicana (Fig. 1).

Representatividad de Crotalus en las ANP. En la actualidad existen en México 173 ANP con decreto federal (CONANP, 2010), de las cuales 146 (84.4\%) están ubicadas en áreas donde se estima la distribución al menos de 1 especie del género Crotalus. Asimismo, de las 34 especies de Crotalus que se distribuyen en México, se calcula que 30 (88.2\%) están presentes al menos dentro de 1 ANP (Cuadro 2). Las 


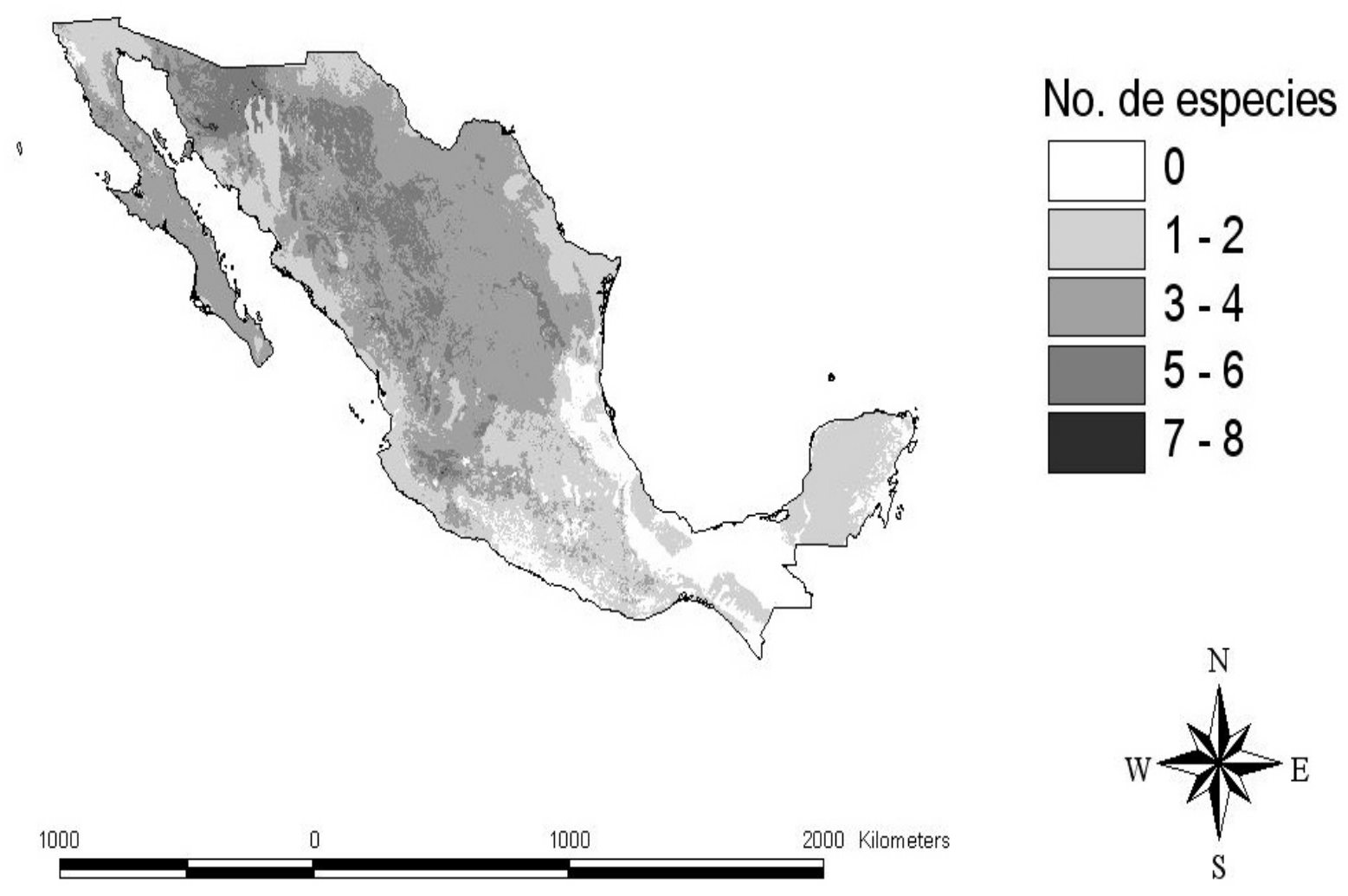

Figura 1. Patrón de distribución de la riqueza de especies del género Crotalus en México.

4 especies que no se encuentran incluidas en ninguna ANP son C. caliginis, C. ericsmithi, C. lannomi, y C. stejnegeri. Las mejor representadas en el esquema de las ANP son $C$. molossus y C. simus, presentes en 67 y 60 ANP respectivamente. Otras de las especies con alta representatividad son C. scutulatus, C. atrox y C. lepidus presentes en 55,40 y 35 ANP, respectivamente (Cuadro 2). Se encontró que existe una correlación importante entre el número de las ANP donde está presente una determinada especie y la extensión de su área de distribución $(r=0.874, P<0.0001)$.

De las 21 especies endémicas de México, se estima que C. caliginis, C. ericsmithi, C. lannomi, y C. stejnegeri no están en ninguna ANP. Por otra parte, de las 31 especies dentro de alguna categoría de la Lista Roja de la IUCN (IUCN, 2010), todas están incluidas excepto C. caliginis y C. stejnegeri, y con excepción de C. stejnegeri, todas las especies incluidas en la NOM-059-SEMARNAT-2010 (SEMARNAT, 2010) están presentes en al menos 1 ANP. Sin embargo, es importante enfatizar que dado el rezago que tuvo la publicación de dicha Norma Oficial y los cambios en la nomenclatura y taxonomía de este grupo de serpientes, ya se hace necesaria la actualización de esta Norma.

El ANP que potencialmente contienen más especies de Crotalus es el Área de Protección de Flora y Fauna Islas del Golfo de California, con 9 especies, seguida de las Áreas de Protección de Flora y Fauna Corredor Biológico Chichinautzin y Ajos/Bavispe, el Área de Protección de Recursos Naturales Cuencas de los Ríos Valle de Bravo, Malacatepec, Tilostoc y Temascaltepec; los Parques Nacionales Cumbres de Majalca e Insurgente José María Morelos, y las Reservas de la Biosfera Barranca de Metztitlán, Sierra de Manantlán y Sierra Gorda, con 7 especies cada una (Cuadro 2).

\section{Discusión}

Sobre la precisión de los resultados. Los errores de comisión detectados en este estudio pueden deberse a diferentes razones; una es que la información presentada por Cambell y Lamar (2004) carezca de un registro completo de las especies en dichas zonas y que efectivamente las especies 
estén presentes ahí; otra posible razón es que los mapas generados representen el nicho ecológico fundamental de las especies (Soberón y Peterson, 2005), pero que los factores históricos (barreras geográficas, capacidad de dispersión limitada) o bióticos (competencia, depredación) hayan impedido que esas especies ocupen las áreas previstas, o bien, que estén extintas (Anderson et al., 2003; Soberón y Peterson, 2005).

Los errores de omisión pueden deberse a que el modelo generado no sea preciso debido a que los registros fueron poco representativos, a errores en la identificación de la especie, al proceso de georreferenciación empleado, o bien, a que el registro represente individuos fuera de su distribución nativa (Anderson et al., 2003). No obstante, en algunos de los modelos existen pequeñas áreas de presencia de las especies correspondientes, lo que indica que no están ausentes del todo pero que probablemente por la fragmentación histórica o reciente de su hábitat, su distribución ha perdido continuidad en estas zonas generando poblaciones aisladas.

El uso de GARP como herramienta para predecir la distribución potencial de especies mediante el modelado de su nicho ecológico tiene como desventaja su sistemática sobre-predicción espacial (Elith y Graham, 2006). Esto podría ser particularmente inadecuado en un estudio como éste, ya que al sobreestimar la distribución potencial de las especies de Crotalus, se generaría una sobreestimación de su representatividad en las ANP. Sin embargo, a diferencia de lo encontrado por Elith y Graham (2006), quienes colocan a MaxEnt entre los algoritmos con mejor desempeño cuando se trabaja con paisajes adecuadamente muestreados, se ha encontrado que en regiones pobremente estudiadas el poder predictivo de GARP y MaxEnt no son significativamente diferentes; incluso, MaxEnt tiene poca capacidad para predecir distribuciones en regiones amplias y poco muestreadas (Peterson et al., 2007). Así, dada la escala amplia de este estudio y que la información disponible sobre registros geográficos para varias de las especies de Crotalus en México es escasa, el uso del algoritmo GARP fue adecuado en la mayoría de los casos (65.2\%). No obstante, es posible que en algunas de las especies para las que se obtuvo una cantidad adecuada de registros y con buena representatividad exista un cierto nivel de sobrepredicción en su distribución. Cualquier modelo predictivo de distribución de especies está limitado porque no se conoce con certeza la distribución real de las especies; por lo tanto, sólo es posible evaluar el desempeño matemático del modelo y no necesariamente el nivel de representación de la distribución real de las especies. De cualquier modo, el desarrollo y uso de modelos predictivos de distribución de especies se ha incrementado en los últimos años, ya que es la herramienta disponible que permite estimar lo que se puede encontrar en la realidad (Martínez-Meyer et al., 2004). Por otra parte, la calidad de los datos, en términos de precisión y representatividad, también es un factor relevante al trabajar con modelos predictivos de distribución de especies, ya que de ello depende, en buena medida, la aproximación de las predicciones a la realidad (Anderson et al., 2003). Aunque en este estudio los registros para algunas de las especies eran pocos, en varios casos fueron suficientes para representar su área de distribución y generar modelos relativamente confiables (Lindermayer et al., 1996; Peterson et al., 1999; Hirzel et al., 2001) y muy similares a los mapas de distribución propuestos en los trabajos de Grismer (2002) y Campbell y Lamar (2004).

Patrón de riqueza de las especies de Crotalus en México. La mayor riqueza de crotalinos en México no se concentra únicamente en la Faja Volcánica Transmexicana, considerada como una área de importancia en riqueza de especies de reptiles y anfibios (Ochoa-Ochoa y Flores Villela, 2006). Para las serpientes de cascabel, la sierra Madre Occidental, el noroeste del Altiplano Mexicano y la mitad norte de la sierra Madre Oriental mostraron ser regiones con una alta riqueza de especies (más de 4 especies por $\mathrm{km}^{2}$ ); así como la península de Baja California, incluyendo sus islas, identificada como una región de gran importancia no sólo en términos de riqueza de especies sino también por sus numerosos endemismos asociados $(C$. angelensis, $C$. caliginis, C. catalinensis, C. estebanensis, C. lorenzoensis, C. muertensis y C. tortuguensis) (Grismer, 2002; ParedesGarcía, 2008). En contraste, se calcula que la riqueza de especies es menor ( 1 o 2 especies por $\mathrm{km}^{2}$ ) en el sur del país, en la región del golfo de México y en la costa del Pacífico mexicano (Fig. 1).

Representatividad de las especies de Crotalus en las ANP e implicaciones para su conservación. La gran variedad de ambientes en los que habita el género Crotalus incide en que esté ampliamente distribuido en México, ocupando prácticamente todo el territorio (Paredes-García, 2008) y que en consecuencia, la mayor parte de sus especies se encuentre dentro de alguna ANP. Sin embargo, a pesar de que la representatividad de crotalinos en las ANP de México es alta, es importante enfatizar que las 4 especies que no están incluidas en ninguna ANP son especies endémicas de México y de distribución restringida (Grismer, 2002; Campbell y Lamar, 2004; Campbell y Flores-Villela, 2008); además, 2 de ellas (C. lannomi y C. stejnegeri) presentan un estado de conservación delicado (Cuadro 1). En este contexto de alta representatividad de las especies del género Crotalus dentro de las ANP, la idea de plantear la creación de áreas adicionales para la protección y conservación de crotalinos se dificulta, más aún, cuando las especies no incluidas en las ANP tienen una distribución muy localizada y están presentes en áreas distantes entre 
sí y de alguna ANP. Por esta razón, y porque estas especies son un componente importante de la biodiversidad, es fundamental incluirlas en los esfuerzos de conservación para enfrentar las amenazas que las afectan. Las estrategias alternativas para su conservación deben incluir, entre otras, la educación ambiental, la conservación de su hábitat mediante el uso sustentable de recursos, el manejo y la reproducción en cautiverio de especies en riesgo (Zug et al., 2001). Asimismo, es necesario que en las áreas donde se distribuyan estas especies y donde se predice su presencia, se realicen estudios de campo que permitan conocer más sobre su distribución y sus requerimientos ecológicos (Zug et al., 2001; Lobo y Hortal, 2003; Raxworthy et al., 2003). Estos estudios también podrían contribuir a conocer mejor la biodiversidad de estos sitios a fin de determinar si en estas mismas localidades habitan otras especies de interés y con problemas de conservación. De esta manera, se reforzaría el conocimiento biológico de estas zonas que probablemente puedan constituir áreas con una significativa riqueza de especies y de endemismos, lo que finalmente podría promover el establecimiento de ANP adicionales (Challenger, 1998).

Aún es parcial, o incluso nulo, el conocimiento de la historia natural de muchas de las especies de este grupo; además, hace falta sensibilizar a la sociedad en la conservación de sus poblaciones y hábitats. La falta de conocimiento y sensibilización se refleja en las consecuencias negativas que tienen las actividades humanas que se traducen en la destrucción y fragmentación de sus ambientes, aunado a su eliminación directa (Campbell y Lamar, 2004). Estos son algunos de los factores más importantes que inciden en la pérdida de diversidad local y regional de crotalinos (Campbell y Lamar, 2004).

Es necesario que en el corto plazo se realicen estudios que complementen la información aquí presentada, con el fin de incrementar el conocimiento de estas especies para tomar mejores decisiones y plantear estrategias de manejo más adecuadas para mantener su diversidad. Al ser México el país que alberga la mayor diversidad de especies del género Crotalus, debe asumir un fuerte compromiso moral y activo con el fin de realizar mayores y mejores esfuerzos para disminuir las amenazas que afectan estas especies.

\section{Agradecimientos}

Este estudio fue financiado a través de los proyectos "Diversidad biológica del estado de Hidalgo", FomixHgo-2006-43761; PROMEP, Cuerpo Académico de Ecología, y CONACYT S52552-Q. Agradecemos a Sandra Berman por la georreferenciación de los numerosos registros. Se agradece a los revisores anónimos los atinados comentarios hechos a este trabajo.

\section{Literatura citada}

Alvarado-Díaz, J. y J. A. Campbell. 2004. A new montane rattlesnake (Viperidae) from Michoacán, México. Herpetologica 60:281-286.

Anderson, R. P., D. Lew y A. T. Peterson. 2003. Evaluating predictive models of species' distributions: criteria for selecting optimal models. Ecological Modelling 162:211-232.

Campbell, J. A. y O. Flores-Villela. 2008. A new longtailed rattlesnake (Viperidae) from Guerrero, México. Herpetologica 64:246-257.

Campbell, J. A. y W. W. Lamar. 1989. The venomous reptiles of Latin America. Comstock/ Cornell University Press, Ithaca, New York. 425 p.

Campbell, J. A. y W. W. Lamar. 2004. The venomous reptiles of the western hemisphere, vol. II. Comstock/ Cornell University Press, Ithaca, New York. 870 p.

Castellón-Huerta, B. 2001. Cúmulo de símbolos. La serpiente emplumada. Arqueología Mexicana 53:28-35.

Challenger, A. 1998. Utilización y conservación de los ecosistemas terrestres de México. Pasado, presente y futuro. Comisión Nacional para el Conocimiento y Uso de la Biodiversidad/Instituto de Biología, UNAM/Agrupación Sierra Madre, México, D. F. 847 p.

CONABIO (Comisión Nacional para el Conocimiento y Uso de la Biodiversidad). 1998. Topografía de México. Escala 1:250,000. Extraído del modelo digital del terreno. http:// conabioweb.conabio.gob.mx/metacarto/metadatos.pl; última consulta: 05.IX.2008.

CONABIO (Comisión Nacional para el Conocimiento y Uso de la Biodiversidad). 1999. Uso de suelo y vegetación modificado por CONABIO, escala 1:1, 000,000. http://conabioweb. conabio.gob.mx/metacarto/metadatos.pl; última consulta: 09.I.2008.

CONANP (Comisión Nacional de Áreas Naturales Protegidas). 2010. Áreas Naturales Protegidas Federales de México y Áreas Destinadas Voluntariamente a la Conservación. Morelia, Michoacán. http://www.conanp.gob.mx/sig/ informacion/info.htm; última consulta: 02.II.2010.

Elith, J. y C. H. Graham. 2006. Novel methods improve prediction of species' distributions from occurrence data. Ecography 29:129-151.

Flores-Villela, O. 1993. Herpetofauna mexicana. The Carnegie Museum of Natural History, Pittsburg, Pennsylvania. 73 p.

Flores-Villela, O. y L. Canseco-Márquez. 2004. Nuevas especies y cambios taxonómicos para la herpetofauna de México. Acta Zoológica Mexicana (n.s.) 20:115-144.

García, E. y CONABIO (Comisión Nacional para el Conocimiento 
y Uso de la Biodiversidad). 1998. Climas (clasificación de Köppen, modificada por García). Escala 1:1,000,000. http:// conabioweb.conabio.gob.mx/metacarto/metadatos.pl; última consulta: 06.II.2008.

GBIF (Global Biodiversity Information Facility). 2008. Crotalus spp. http://data.gbif.org/species/; última consulta: 31.V.2009.

Grismer, L. L. 2002. The amphibians and reptiles of Baja California, its Pacific islands, and the islands in the Sea of Cortés: natural history, distribution and identification. University of California Press, Berkeley. 409 p.

Hijmans, R. J., S. Cameron y J. Parra. 2004. WorldClim v. 1.2. Museum of Vertebrate Zoology, University of California, Berkeley. http://biogeo.berkeley.edu/worldclim/worldclim. htm; última consulta: 05.VI.2009.

Hijmans, R. J., S. E. Cameron, J. L. Parra, P. G. Jones y A. Jarvis. 2005. Very high resolution interpolated climate surfaces for global land areas. International Journal of Climatology 25:1965-1978.

Hirzel, A. H., V. Helfer y F. Metral. 2001. Assessing habitatsuitability models with a virtual species. Ecological Modelling 145:111-121.

IUCN (International Union for Conservation of Nature). 2010. IUCN red list of threatened species. Version 2010.4. http:// www.iucnredlist.org; última consulta: 28.V.2011.

Jáuregui, J. 2001. La serpiente emplumada entre los coras y huicholes. Arqueología Mexicana 53:28-35.

Lindenmayer, D.B., B.G. Mackey y H.A. Nix. 1996. The bioclimatic domains of four species of commercially important eucalypts from south-eastern Australia. Australian Forest.ry Journal 59:74-89.

Lobo, J. M. y J. Hortal. 2003. Modelos predictivos: un atajo para describir la distribución de la diversidad biológica. Ecosistemas 2003. http//www.aeet.org/ecosistemas/031/ investigacion3; última consulta: 27.IX.2008.

Martínez-Meyer, E., A. T. Peterson y W. W. Hargrove. 2004. Ecological niches as stable distributional constraints on mammal species, with implications for Pleistocene extinctions and climate change projections for biodiversity. Global Ecology and Biogeography 13:305-314.

Ochoa-Ochoa, L. M. y O. Flores-Villela. 2006. Áreas de diversidad y endemismo de la herpetofauna mexicana. Universidad Nacional Autónoma de México/ Comisión Nacional para el Conocimiento y Uso de la Biodiversidad, México, D. F. 211 p.

Paredes-García, D. M. 2008. Distribución de especies de Crotalus en México mediante modelos predictivos de distribución.
Tesis, Universidad Autónoma del Estado de Hidalgo, Pachuca, Hidalgo. 73 p.

Peterson, A. T., J. Soberón y V. Sánchez-Cordero. 1999. Conservatism of ecological niches in evolutionary time. Science 285:1265-1267.

Peterson, A. T., M. Papes y M. Eaton. 2007. Transferability and model evaluation in ecological niche modeling: a comparison of GARP and MaxEnt. Ecography 30:550-560.

Phillips, S. J., R. P. Anderson y R. E. Schapire. 2006. Maximum entropy modeling of species geographic distributions. Ecological Modelling, 190:231-259.

Raxworthy, C. J., E. Martínez-Meyer, N. Horning, R. A. Nussbaum, G. E. Schneider, M. A. Ortega-Huerta y A. T. Peterson. 2003. Predicting distributions of known and unknown reptile species in Madagascar. Nature 426:837-841.

Scachetti-Pereira, R. 2002. Desktop GARP v. 1.1.6. The University of Kansas Biodiversity Research Center and Reference Center for Environmental Information. http:// www.nhm.ku.edu/desktopgarp/index.html; última consulta: 12.XI.2007.

SEMARNAT (Secretaría de Medio Ambiente y Recursos Naturales). 2010. Norma Oficial Mexicana NOM-059SEMARNAT-2010, Protección ambiental - Especies nativas de México de flora y fauna silvestres - Categorías de riesgo y especificaciones para su inclusión, exclusión o cambio Lista de especies en riesgo. Diario Oficial de la Federación, segunda sección; 30 de diciembre: 1-78.

Soberón, J. y A. T. Peterson. 2005. Interpretation of models of fundamental ecological niches and species' distributional areas. Biodiversity Informatics 2:1-10.

Stockwell, D. R. B. y D. Peters. 1999. The GARP modeling system: problems and solutions to automated spatial prediction. International Journal of Geographic Information Science 13:143-158.

Stockwell, D. R. B. y I. R. Noble. 1992. Induction of sets of rules from animal distribution data: a robust and informative method of data analysis. Mathematics and Computers in Simulation 33:385-390.

Taube, K. A. 2001. La serpiente emplumada en Teotihuacan. Arqueología Mexicana 53:36-41.

Valencia, H. A. A. 2006. Taxonomía y distribución del género Crotalus (Linneo, 1758) en el estado de Hidalgo. Tesis, Universidad Autónoma del Estado de Hidalgo, Pachuca. 104 p.

Zug, G. R., L. J. Vitt y J. P. Caldwell. 2001. Herpetology. An introductory biology of amphibians and reptiles, segunda edición. Academic, New York. 630 p. 